\title{
Prenatal exposure to organophosphate pesticides and brain morphology and white matter microstructure in preadolescents
}

\author{
Michiel A. van den Dries ${ }^{\mathrm{a}, \mathrm{b}}$, Sander Lamballais ${ }^{\mathrm{b}, \mathrm{c}, \mathrm{d}}$, Hanan El Marroun ${ }^{\mathrm{a}, \mathrm{e}, \mathrm{f}}$, Anjoeka Pronk ${ }^{\mathrm{g}}$, \\ Suzanne Spaan ${ }^{g}$, Kelly K. Ferguson ${ }^{\mathrm{h}}$, Matthew P. Longnecker ${ }^{\mathrm{i}}$, Henning Tiemeier ${ }^{\mathrm{a}, \mathrm{j}}$, \\ Mònica Guxens ${ }^{a, k, l, m, "}$ \\ ${ }^{\text {a }}$ Erasmus MC, University Medical Center Rotterdam, Department of Child and Adolescent Psychiatry, Rotterdam, 3015 CN, the Netherlands \\ ${ }^{\mathrm{b}}$ Erasmus MC, University Medical Center Rotterdam, The Generation R Study Group, Rotterdam, 3015 CN, the Netherlands \\ ${ }^{\mathrm{c}}$ Erasmus MC, University Medical Center Rotterdam, Department of Epidemiology, Rotterdam, 3015 CN, the Netherlands \\ ${ }^{\mathrm{d}}$ Erasmus MC, University Medical Center Rotterdam, Department of Clinical Genetics, Rotterdam, 3015 CN, the Netherlands \\ e Erasmus MC, University Medical Center Rotterdam, Department of Pediatrics, Rotterdam, 3015 CN, the Netherlands \\ ${ }^{\mathrm{f}}$ Department of Psychology, Education and Child Studies, Erasmus School of Social and Behavioral Sciences, Erasmus University Rotterdam, 3062 PA, the Netherlands \\ ${ }^{g}$ Department of Risk Analysis for Products in Development, TNO, Utrecht, 3584 CB, the Netherlands \\ ${ }^{\mathrm{h}}$ Epidemiology Branch, National Institute of Environmental Health Sciences, National Institutes of Health, Department of Health and Human Services, Durham, North \\ Carolina, NC, 27709, USA \\ ${ }^{\mathrm{i}}$ Ramboll, Raleigh, North Carolina, NC, 27612, USA \\ ${ }^{\mathrm{j}}$ Department of Social and Behavioral Sciences, Harvard T. H. Chan School of Public Health, Boston, MA, 02115, USA \\ ${ }^{\mathrm{k}}$ ISGlobal, Barcelona, 08003, Spain \\ ${ }^{1}$ Pompeu Fabra University, Barcelona, 08002, Spain \\ ${ }^{\mathrm{m}}$ Spanish Consortium for Research on Epidemiology and Public Health (CIBERESP), Instituto de Salud Carlos III, 28029, Spain
}

\section{A R T I C L E I N F O}

\section{Keywords:}

Organophosphate pesticides

Prenatal exposure

MRI

White matter microstructure

Brain morphology

\begin{abstract}
A B S T R A C T
Background: Prenatal exposure to organophosphate (OP) pesticides associate with impaired neurodevelopment in humans and animal models. However, much uncertainty exists about the brain structural alterations underlying these associations. The objective of this study was to determine whether maternal oP pesticide metabolite concentrations in urine repeatedly measured during gestation are associated with brain morphology and white matter microstructure in 518 preadolescents aged 9-12 years.

Method: Data came from 518 mother-child pairs participating in the Generation R Study, a population-based birth cohort from Rotterdam, the Netherlands. Maternal urine concentrations were determined for 6 dialkylphosphates (DAPs) including 3 dimethyl (DM) and 3 diethyl (DE) alkyl phosphate metabolites, collected at early, mid, and late pregnancy. At child's age 9-12 years, magnetic resonance imaging was performed to obtain T1-weighted images for brain volumes and surface-based cortical thickness and cortical surface area, and diffusion tensor imaging was used to measure white matter microstructure through fractional anisotropy (FA) and mean diffusivity (MD). Linear regression models were fit for the averaged prenatal exposure across pregnancy.

Results: DM and DE metabolite concentrations were not associated with brain volumes, cortical thickness, and cortical surface area. However, a 10-fold increase in averaged DM metabolite concentrations across pregnancy was associated with lower FA $(\mathrm{B}=-1.00,95 \% \mathrm{CI}=-1.80,-0.20)$ and higher $\mathrm{MD}(\mathrm{B}=0.13,95 \% \mathrm{CI}=0.04$, 0.21). Similar associations were observed for DE concentrations.

Conclusions: This study provides the first evidence that OP pesticides may alter normal white matter microstructure in children, which could have consequences for normal neurodevelopment. No associations were observed with structural brain morphology, including brain volumes, cortical thickness, and cortical surface area.
\end{abstract}

\footnotetext{
* Corresponding author. Barcelona Institute for Global Health - Campus Mar, Doctor Aiguader, 88, 08003, Barcelona, Spain.

E-mail address: monica.guxens@isglobal.org (M. Guxens).
} 


\section{Introduction}

Organophosphate (OP) pesticides are chemical agents often used in agriculture to protect crops against insects. At present, five billion pounds of pesticides are being applied worldwide and approximately $33 \%$ are OP pesticides (Mahajan et al., 2019). Similarly, between 1998 and 2008 one third of the insecticides used in the Netherlands were OP pesticides (CBS, 2020). In the past decade, the use of OP pesticides has been declining in both the Netherlands and the European Union (EU) due to stricter legislations. However, several OP pesticides such as malathion are currently approved by the EU and OP pesticide residues are frequently detected on tested vegetables and fruits coming from importation (ChemKap, 2017; EU Pesticides database, 2020).

Since OP pesticide residues may persist on or in food after crop harvesting (Eaton et al., 2008), there is an increasing concern about their potential harmful health effects. The exposure to OP pesticides generally occurs through the consumption of food (Lu et al., 2008). However, residential exposure can also occur through the use of insecticides in and around the house or by living in close proximity to agricultural lands were OP pesticides are being applied (Fenske et al., 2002; Julien et al., 2007; Lu et al. 2000, 2004; Valcke et al., 2006; Whyatt et al., 2003).

It is well established that the exposure to high concentrations of OP pesticides is neurotoxic to both humans and animals (Costa et al., 2008; Eaton et al., 2008). However, evidence exist that OP pesticide exposure at fairly low-dose levels may also have a negative health effect (Savy et al., 2018; Slotkin et al., 2008). OP pesticides are able to pass the placental and the blood-brain barrier (Bradman et al., 2003) and, during gestation, the development of the human brain is especially susceptible to neurotoxic effects (Rice and Barone, 2000). Therefore, pregnancy exposure to low-dose levels of OP pesticides might affect fetal normal brain development.

Although several epidemiological studies have reported associations between pregnancy OP pesticide exposure and offspring's neuropsychological development (Sapbamrer and Hongsibsong, 2019), much uncertainty exists about the brain structural alterations underlying these associations. Magnetic resonance imaging (MRI) is a useful instrument for addressing these knowledge gaps and can help identify the associations between neurotoxic exposures and brain development (Rauh and Margolis, 2016). In humans, altered brain morphology and white matter microstructure is associated with impaired cognition, behavior problems, and neurodevelopmental disorders (Dennis and Thompson, 2013; Gilmore et al., 2018; Lebel and Deoni, 2018; Mizuno et al., 2019). So far, only few animal studies and one small epidemiological study have investigated the effect of OP pesticide exposure on morphological brain measures. Experimental animal studies showed that OP pesticide exposure was associated with smaller brain volumes, both thinning and thickening of the cortex, and alterations of white matter microstructure (Mullins et al., 2015; Roy et al. 2004, 2005). In humans, prenatal exposure to the OP pesticide chlorpyrifos measured in cord blood was associated with thinner cortices and alterations in cortical surface area in 40 children at 6-11 years of age (Rauh et al., 2012). However, this previous human study only analyzed a specific OP pesticide, was restricted to a small sample size, and was unable to investigate the exposure across the entire pregnancy. Moreover, no previous epidemiological study investigated the association between prenatal OP pesticide exposure and white matter microstructure, which has been observed in a previous animal study (Mullins et al., 2015).

Therefore, the objective of this study was to determine whether maternal OP pesticide metabolite concentrations in urine repeatedly measured during gestation are associated with brain morphology and white matter microstructure in 518 preadolescents at 9-12 years of age. Understanding the association between prenatal OP pesticide exposure and brain morphology and white matter microstructure may help explain the association between pregnancy OP pesticide exposure and offspring's neuropsychological development observed in previous studies. Further, findings of the present study may assist in future policies regarding the regulation of OP pesticide application.

\section{Materials and methods}

\subsection{Study population and follow-up}

This research was embedded in the Generation $\mathrm{R}$ Study, a population-based cohort from early fetal life onwards in Rotterdam, the Netherlands, which has been described in detail previously (Kooijman et al., 2016). Fig. S1 presents a flowchart of this study. Briefly, all pregnant women who lived in the study area in Rotterdam, the Netherlands and were expected to have a delivery between 2002 and 2006 were eligible. A total of 8879 women were enrolled during pregnancy. A random sample of 800 mother-child pairs were selected for assessment of OP pesticide metabolites among the 1449 that provided three spot urine samples during pregnancy and had child's neurodevelopmental data at postnatal visits. Of those, 518 children were included in the present study as they had good quality data on MRI measurements at 9-12 years of age. Human subjects review for the procedure of this study was carried out and approved by the Medical Ethics Committee of the Erasmus Medical Center, Rotterdam (IRB Registration no.: IRB00001482, MEC-2012-165, MEC-2007-413, MEC, 217.595/2002/202, and MEC 198.782.2001.31). Written informed consent for the children and mothers was provided by the mothers.

\subsection{Urine collection and analysis of $O P$ pesticide metabolites}

A more detailed description of urine specimen collection and measurement of $\mathrm{OP}$ pesticide metabolites have been published previously (van den Dries et al., 2018) and can be found in the supplement (Methods S1). Briefly, 6 non-specific urinary dialkylphosphate (DAP) metabolites of OP pesticides were measured from urine samples collected at $<18,18-25$, and $>25$ weeks of gestation by gas chromatography coupled with tandem mass spectrometry (GC-MS/MS). These include 3 dimethyl alkyl phosphate (DM) and 3 diethyl alkyl phosphate (DE) metabolites. Creatinine concentrations were also measured in order to correct for urinary dilution. All urine samples had detectable concentrations of most metabolites. The intraclass correlation of DAP metabolite concentrations was weak for a single concentration (0.22-0.26) and moderate for the average of the 3 concentrations (0.51-0.54) (van den Dries et al., 2019).

\subsection{Magnetic resonance imaging}

Details of the neuroimaging acquisition and processing can be found in the supplemental material (Methods S2). The global brain metrics derived from $\mathrm{T}_{1}$-weighted images included total brain volume, cerebral and cerebellar white and grey matter volume, and subcortical grey matter volume. Additionally, we focused on the corpus callosum and the subcortical regions: amygdala, caudate nucleus, hippocampus, pallidum, putamen, nucleus accumbens and the thalamus (Muetzel et al., 2019). Surface-based thickness and surface area maps were made of the cerebral cortex (Muetzel et al., 2019). Diffusion tensor imaging (DTI) was used to fit diffusion tensors at each voxel and fractional anisotropy (FA) and mean diffusivity (MD) were computed (Cook et al., 2006). Twelve major white matter tracts were identified via probalistic tractography with the FSL plugin AutoPtx (de Groot et al., 2015; Muetzel et al., 2017). These included the forceps minor and major, and the bilateral tracts of the cingulum bundle, corticospinal tract, the inferior and superior longitudinal fasciculi, and the uncinate fasciculus. The mean FA and MD per tract, weighted by the connectivity distribution, were then computed. A confirmatory factor analysis was performed to model a single latent FA and MD measure across the 12 tracts, which represented global FA and MD across the brain (Muetzel et al., 2015). Global FA indicates the tendency for preferential water diffusion in 
white matter tracts. A lower FA score indicates in general that the comprising axons are less densely packed and the directionality of the water diffusion is not uniformly directed as compared with well-organized tracts. Global MD describes the magnitude of average water diffusion in all directions within brain tissue, with higher values generally occurring in white matter tracts that show a less well-organized structure (Alexander et al., 2007; Lubczyńska et al., 2020).

\subsection{Potential confounders}

Potential adjustment variables were selected a priori defined as the minimal sufficient adjustment set with a Directed Acyclic Graph (DAG) using the Dagitty software (Textor et al., 2017). The DAG was based on previous studies of prenatal OP pesticide exposure and neurodevelopment and on biologically plausible covariate-exposure and covariate-outcome associations observed in our data (see Fig. S2). We further included adjustment variables that are ancestors of the exposure and ancestors of the outcome to increase precision. The adjustment variables were household income [less than 1200 euro/month (i.e., less than the social security level of the Netherlands), 1200-2000 euro/month, more than 2000 euro/month], maternal highest achieved level of education [low (less than 3 years of high school), intermediate (3 or more years of secondary education), and high (university degree or higher vocational training)], maternal ethnical background (Dutch, other Western, and non-Western), maternal age at enrolment, marital status (married/living with partner versus single), maternal parity $(0,1$, or 2 or more children), maternal smoking habits during pregnancy (none, only until pregnancy known, or continued after pregnancy known), maternal gestational alcohol use [none, only until pregnancy known, continued infrequently ( $<1$ glass/week) or continued regularly ( $\geq 1$ glass/week)], maternal pre-pregnancy body mass index (BMI) $\left(\mathrm{kg} / \mathrm{m}^{2}\right)$, maternal IQ (assessed when the mother-child pairs visited the research center for the 6-year examination and measured by using the computerized Ravens Advanced Progressive Matrices Test, set I (Prieler, 2003)), child sex, and the child age at the MRI scan.

\subsection{Statistical methods}

Total DM (nmol/1) was created by summing dimethylphosphate, dimethylthiophosphate, and dimethyldithiophosphate metabolite concentrations. Total DE (nmol/l) was defined as the sum of diethylphosphate, diethylthiophosphate, and diethyldithiophosphate metabolite concentrations. Total DAP (nmol/l) was created by summing the 6 metabolites. These concentrations were creatinine adjusted (nmol/ $\mathrm{g}$ creatinine) and transformed using a log transformation (base 10) to improve linearity of the dose-response relation and model fit. Few concentrations were missing because of an inadequate sample or machine error. We therefore imputed missing concentrations $(<1 \%)$ and missing confounder information 10 times by using the Multivariate Imputation by Chained Equations (MICE) package in R (R core Team, 2015; van Buuren and Groothuis-Oudshoorn, 2011). We included total brain volume and global FA in the imputation procedure as predictors, but we did not impute them.

As a first step, we applied linear regression to examine the association of averaged DM, DE, and DAP concentrations over pregnancy with brain volumes. To account for multiple testing (14 tests for each exposure), we applied the false discovery rate (FDR) correction. As a second step, we investigated metabolite concentrations - brain volume associations for each exposure time point (early, mid, and late pregnancy) separately. We performed this second step for the identification of possible periods of susceptibility and to have the ability to compare our findings with studies that only used one urine sample during gestation to measure the exposure to OP pesticides. These analyses were also corrected for multiple testing using the FDR correction. The same multiple linear regressions were applied for the DAP - global white matter tract
(FA and MD) associations. Post-hoc analyses were run on the 12 major individual white matter tracts when the analysis yielded a significant association between prenatal DAP metabolite concentrations and global white matter tracts. We explored whole-brain vertex-wise statistics using the QDECR R package (https://qdecr.com) for total DM, DE, and DAP metabolite concentrations in association with local cortical thickness and cortical surface area. Vertex-wise analyses were corrected for multiple testing by the application of Gaussian Monte Carlo null-Z simulations (The cluster-forming threshold defined as $\mathrm{p}<0.001$ ). Next, these analyses were also corrected by applying a Bonferroni adjustment for the analyses of both hemispheres.

All models were adjusted for potential confounders described above. Additionally, we adjusted models of subcortical and cerebellar volumes for intracranial volume to ascertain relativity to head size. Models of the other volumes were not adjusted for intracranial volume as they were highly correlated (between $r=0.81$ and $r=0.93$ ).

As sensitivity analyses, first, we investigated potential effect modification by sex via interaction terms $(P$-value for interaction $<0.05)$ to compare our results with previous studies who observed sex specific effects (Rauh et al., 2012; Sagiv et al., 2019). Second, we applied inverse probability weighting to adjust all models for loss to follow-up and to deal with potential selection bias because participants included in this study were older, had higher educational level, and more frequently Dutch as compared to the complete Generation R Study cohort (van den Dries et al., 2018). Third, because diet and the intake of healthy nutrients may confound the association between prenatal OP pesticide exposure (e.g., residues on fruits) (van den Dries et al., 2018) and brain development (e.g., healthy nutrients) (Fig. S2), we performed a sensitivity analyses in which we additionally adjusted for maternal fruit and vegetables intake. The consumption of fruit and vegetables was assessed in the first trimester using a modified version of a validated food frequency questionnaire and was adjusted for energy intake (Steenweg-de Graaff et al., 2012).

\section{Results}

\subsection{Descriptive analysis}

The median age of the mothers at enrolment was 31.2 years (IQR $=$ 5.4) and the median age of the child at MRI assessment was 9.8 years $(\mathrm{IQR}=0.3)$ (Table 1$)$. The majority of mothers participating in this study were ethnically Dutch (61.4\%), were nulliparous (66.3\%), were none smokers (79.1\%), had a high educational level (60.2\%), and had a high income (73.6\%). Total DAP metabolite concentrations comprised mostly DM metabolite concentrations (Table 2). The median nmol/g creatinine concentrations were comparable across the three sampling periods. The median total brain volume was $1215 \mathrm{~cm}^{3}\left(\mathrm{IQR}=138 \mathrm{~cm}^{3}\right)$ and median FA was $0.0(\mathrm{IQR}=2.3)$ (Table S1).

\subsection{OP pesticide metabolite concentrations and brain volume}

No associations were observed between averaged maternal DM and DE metabolite concentrations and all brain volumes (Table 3). When specific pregnancy periods were analyzed separately, higher DM and DE metabolite concentrations at $>25$ weeks of gestation were associated with lower thalamus volume, higher DM metabolite concentration at 18-25 weeks of gestation was associated with higher putamen volume, and higher DE metabolite concentrations at $>25$ weeks of gestation were associated with lower cerebellum cortex volume (Table S2). However, these associations did not remain after correction for multiple testing. The results for the total DAP metabolite concentrations were similar to the results observed for the DM and DE metabolite concentrations (Table S3). 
Table 1

Demographic and lifestyle characteristics of 518 mother-child pairs from the Generation R Study population.

\begin{tabular}{|c|c|}
\hline & Median (25th, 75th percentile) or $\%$ \\
\hline \multicolumn{2}{|l|}{ Maternal characteristics } \\
\hline Age & $31.2(28.6,34.0)$ \\
\hline Missing, $n$ & - \\
\hline \multicolumn{2}{|l|}{ Ethnicity } \\
\hline Dutch & $61.4 \%$ \\
\hline Other western & $13.1 \%$ \\
\hline Non-western & $25.5 \%$ \\
\hline Missing, $n$ & - \\
\hline \multicolumn{2}{|l|}{ Educational level } \\
\hline Low & $11.2 \%$ \\
\hline Intermediate & $28.6 \%$ \\
\hline High & $60.2 \%$ \\
\hline Missing, $n$ & 11 \\
\hline \multicolumn{2}{|l|}{ Household income } \\
\hline$<1200$ & $11.6 \%$ \\
\hline $1200-2000$ & $14.8 \%$ \\
\hline$>2000$ & $73.6 \%$ \\
\hline Missing, $n$ & 52 \\
\hline Non-verbal IQ & $100.0(90.0,107.0)$ \\
\hline Missing, $n$ & 7 \\
\hline Body mass index & $23.0(21.2,25.9)$ \\
\hline Missing, $n$ & 2 \\
\hline \multicolumn{2}{|l|}{ Parity } \\
\hline 0 & $66.3 \%$ \\
\hline 1 & $24.2 \%$ \\
\hline$>1$ & $9.5 \%$ \\
\hline Missing, $n$ & 2 \\
\hline \multicolumn{2}{|l|}{ Smoking during pregnancy } \\
\hline No smoking during pregnancy & $79.1 \%$ \\
\hline Until pregnancy recognized & $8.8 \%$ \\
\hline Continued during pregnancy & $12.1 \%$ \\
\hline Missing, $n$ & 40 \\
\hline \multicolumn{2}{|l|}{ Alcohol consumption during pregnancy } \\
\hline No consumption during pregnancy & $33.2 \%$ \\
\hline Until pregnancy recognized & $17.7 \%$ \\
\hline Continued occasionally & $42.1 \%$ \\
\hline Continued frequently & $7.0 \%$ \\
\hline Missing, $n$ & 21 \\
\hline \multicolumn{2}{|l|}{ Child characteristics } \\
\hline Child age at assessment & $9.8(9.6,9.9)$ \\
\hline Missing, $n$ & - \\
\hline \multicolumn{2}{|l|}{ Child sex } \\
\hline Male & $49.4 \%$ \\
\hline Female & $50.6 \%$ \\
\hline Missing, $n$ & - \\
\hline
\end{tabular}

\subsection{OP pesticide metabolite concentrations and white matter microstructure}

Table 3 presents the association between averaged DM and DE metabolite concentrations and white matter microstructure. We observed an association between a 10-fold increase in averaged DM and DE metabolite concentrations and lower FA [B $=-1.00$ (95\%CI: -1.80 , -0.20 ) and $\mathrm{B}=-0.63$ (95\%CI: $-1.24,-0.02)$, respectively]. Next, a 10fold increase in averaged DM and DE metabolite concentrations were associated with higher MD [B $=0.13$ (95\%CI: $0.04,0.21$ ) and $\mathrm{B}=0.06$ (95\%CI: $0.00,0.13)$, respectively]. Regarding the specific pregnancy periods, we observed similar associations for DM and DE concentrations at $<18$ weeks and at $18-25$ weeks of gestation (Table S2). The associations between maternal DAP metabolite concentrations and white matter microstructure were comparable to the results of DM and DE metabolite concentrations (Table S3).

Regarding the individual 12 major white matter tracts, we observed that DM metabolite concentrations averaged across pregnancy were associated with lower FA and higher MD in most of the tracts except for the uncinate fasciculus tract of left hemisphere, the forceps major, and the corticospinal tract of the right hemisphere (Fig. 1 and Table S4). We observed that higher averaged DE metabolite concentrations were associated with lower FA in the superior longitudinal fasciculus tract of the left hemisphere and the corticospinal tracts, and with higher MD in the cingulate gyrus of the cingulum tract of the left hemisphere, the forceps minor tract, and the inferior longitudinal fasciculus tract of the left hemisphere (Fig. 1 and Table S5). The results of the total DAP metabolite concentrations were also almost identical to the results observed for the DM and DE metabolite concentrations (Table S6).

\subsection{OP pesticide metabolite concentrations and cortical thickness and cortical surface area}

We did not find any evidence of an association between prenatal DM, DE, or DAP metabolite concentrations and cortical thickness and cortical surface area using whole-brain vertex-wise analyses (data not shown).

\subsection{Sensitivity analyses}

Effect modification by sex was not observed in the association between averaged DAP metabolite concentrations with cortical and

Table 2

Descriptive statistics of pregnancy DAP metabolite concentrations $(n=518)$.

\begin{tabular}{|c|c|c|c|c|c|c|c|c|c|c|}
\hline & \multicolumn{5}{|c|}{ nmol/g creatinine } & \multicolumn{5}{|c|}{$\mathrm{nmol} / 1$} \\
\hline & $\min$ & p25 & p50 & p75 & $\max$ & $\min$ & p25 & p50 & p75 & $\max$ \\
\hline \multicolumn{11}{|c|}{ DM metabolites in nmol/g creatinine ${ }^{a}$} \\
\hline$<18$ weeks & 6.6 & 153.6 & 255.3 & 420.4 & 6106.5 & 0.9 & 96.1 & 183.4 & 346.7 & 2627.3 \\
\hline 18-25 weeks & 24.8 & 184.2 & 272.1 & 433.6 & 2444.1 & 7.6 & 99.6 & 190.8 & 336.4 & 2396.8 \\
\hline$>25$ weeks & 29.2 & 165.8 & 248.9 & 397.6 & 2857.8 & 10.5 & 103.9 & 194.2 & 326.6 & 3300.5 \\
\hline Averaged & 26.3 & 191.3 & 269.3 & 361.8 & 1381.0 & 14.7 & 118.5 & 179.6 & 289.4 & 1105.1 \\
\hline \multicolumn{11}{|c|}{$\mathrm{DE}$ metabolites in $\mathrm{nmol} / \mathrm{g}$ creatinine ${ }^{\mathrm{b}}$} \\
\hline$<18$ weeks & 0.0 & 25.3 & 46.4 & 86.3 & 3030.5 & 0.0 & 16.0 & 31.3 & 66.2 & 6818.6 \\
\hline 18-25 weeks & 3.3 & 25.2 & 43.4 & 79.6 & 624.3 & 0.6 & 13.9 & 30.1 & 58.4 & 1093.4 \\
\hline$>25$ weeks & 4.1 & 22.0 & 43.9 & 81.5 & 671.4 & 1.1 & 14.7 & 31.5 & 64.4 & 538.2 \\
\hline Averaged & 2.6 & 29.4 & 44.3 & 68.9 & 601.4 & 3.2 & 19.3 & 30.7 & 49.8 & 407.3 \\
\hline \multicolumn{11}{|c|}{ DAP metabolites in nmol/g creatinine ${ }^{c}$} \\
\hline$<18$ weeks & 15.4 & 197.3 & 321.4 & 521.7 & 6444.5 & 6.3 & 119.6 & 224.7 & 422.2 & 7798.7 \\
\hline 18-25 weeks & 41.0 & 222.2 & 323.0 & 519.8 & 2817.0 & 10.0 & 123.2 & 235.9 & 406.1 & 3056.7 \\
\hline$>25$ weeks & 42.1 & 204.1 & 308.0 & 495.7 & 3003.1 & 15.2 & 127.2 & 228.8 & 403.4 & 3332.6 \\
\hline Averaged & 36.7 & 234.6 & 329.9 & 441.2 & 1818.8 & 30.2 & 144.4 & 220.7 & 348.9 & 1259.3 \\
\hline
\end{tabular}

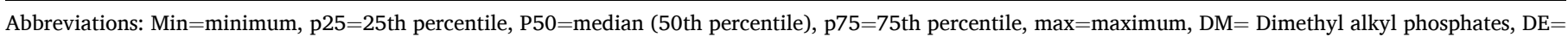
Diethyl alkyl phosphates, $\mathrm{DAP}=$ Total dialkyl phosphates.

a DM is the sum of dimethylphosphate (DMP), dimethylthiophosphate (DMTP), and dimethyldithiophosphate (DMDTP).

b DE is the sum of diethylphosphate (DEP), diethylthiophosphate (DETP), and diethyldithiophosphate (DEDTP).

c DAP is the sum of DMDTP, DMTP, DMP, DEDTP, DETP, and DEP. 
Table 3

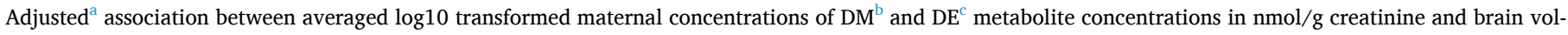
umes $(n=441)$ and white matter microstructure $(n=474)$ assessed at child age 10 years.

\begin{tabular}{|c|c|c|c|c|c|c|c|c|}
\hline \multirow[t]{2}{*}{ Brain volumes } & \multicolumn{4}{|c|}{ Averaged DM metabolite concentrations in $\mathrm{nmol} / \mathrm{g}$ creatinine } & \multicolumn{4}{|c|}{ Averaged DE metabolite concentrations in $\mathrm{nmol} / \mathrm{g}$ creatinine } \\
\hline & B & $95 \% \mathrm{CI}$ & & & B & $95 \% \mathrm{CI}$ & & \\
\hline Total brain & 12.81 & -26.31 & to & 51.92 & 0.22 & -28.98 & to & 29.42 \\
\hline Total grey & 4.69 & -18.75 & to & 28.13 & -3.38 & -20.88 & to & 14.12 \\
\hline Subcortical grey matter & 0.26 & -1.53 & to & 2.04 & -0.69 & -2.03 & to & 0.65 \\
\hline Cerebral white matter & 8.16 & -9.57 & to & 25.90 & 4.27 & -8.98 & to & 17.53 \\
\hline Thalamus ${ }^{\mathrm{d}}$ & -0.36 & -0.74 & to & 0.02 & -0.18 & -0.47 & to & 0.11 \\
\hline Caudate $^{\mathrm{d}}$ & 0.18 & -0.18 & to & 0.55 & -0.11 & -0.38 & to & 0.16 \\
\hline Putamen $^{\mathrm{d}}$ & 0.42 & 0.00 & to & 0.85 & -0.01 & -0.33 & to & 0.31 \\
\hline Pallidum & 0.05 & -0.10 & to & 0.20 & -0.05 & -0.16 & to & 0.06 \\
\hline Hippocampus $^{\mathrm{d}}$ & -0.10 & -0.35 & to & 0.16 & 0.02 & -0.17 & to & 0.21 \\
\hline Amygdala $^{d}$ & 0.05 & -0.08 & to & 0.18 & 0.05 & -0.05 & to & 0.15 \\
\hline Nucleus accumbens ${ }^{\mathrm{d}}$ & 0.00 & -0.07 & to & 0.07 & 0.00 & -0.05 & to & 0.06 \\
\hline Cerebellum cortex ${ }^{\mathrm{d}}$ & -2.68 & -6.51 & to & 1.15 & -2.30 & -5.16 & to & 0.57 \\
\hline Cerebellar white matter & -0.08 & -1.08 & to & 0.91 & -0.46 & -1.20 & to & 0.28 \\
\hline Corpus callosum & -0.06 & -0.27 & to & 0.16 & -0.01 & -0.17 & to & 0.15 \\
\hline \multicolumn{9}{|c|}{ White matter microstructure } \\
\hline Global FA & -1.00 & -1.80 & to & -0.20 & -0.63 & -1.24 & to & -0.02 \\
\hline Global MD & 0.13 & 0.04 & to & 0.21 & 0.06 & 0.00 & to & 0.13 \\
\hline
\end{tabular}

Abbreviations: DM = Dimethyl alkyl phosphates, DE = Diethyl alkyl phosphates, FA = fractional anisotropy, MD = mean diffusivity.

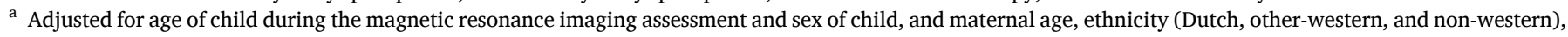

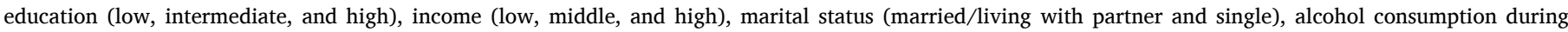

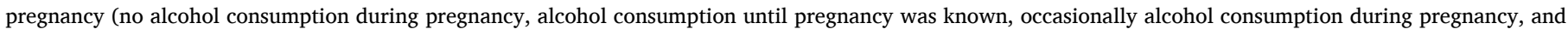

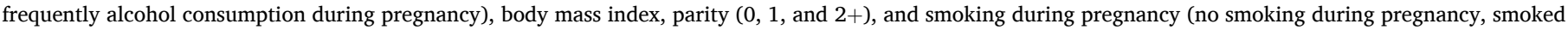
until pregnancy was known, and smoked during pregnancy).

b DM is the sum of dimethylphosphate, dimethylthiophosphate, and dimethyldithiophosphate.

c $\mathrm{DE}$ is the sum of diethylphosphate, diethylthiophosphate, and diethyldithiophosphate.

d Additionally adjusted for intracranial volume.

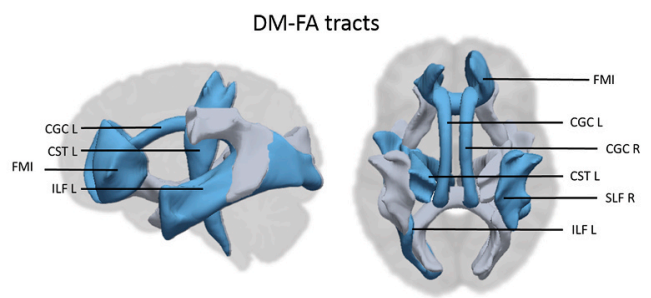

DM-MD tracts
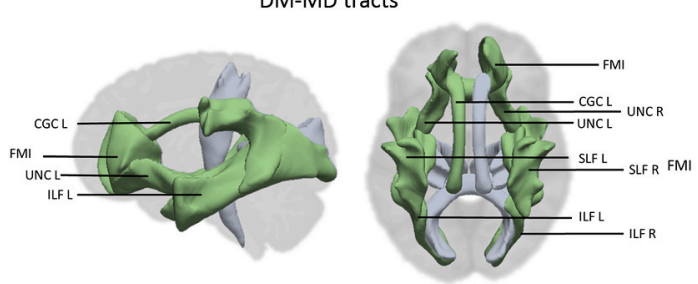

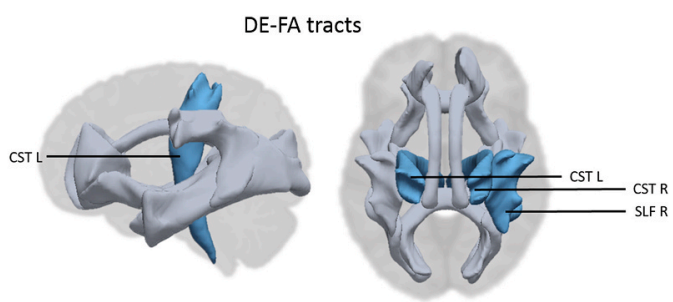

DE-MD tracts
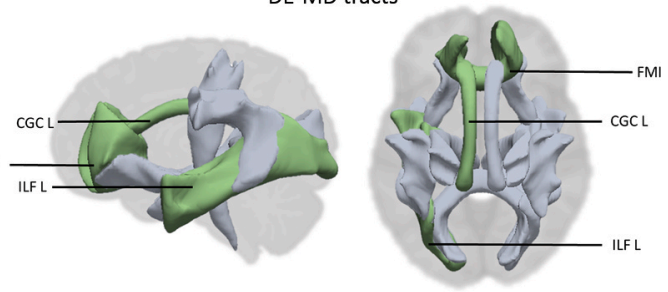

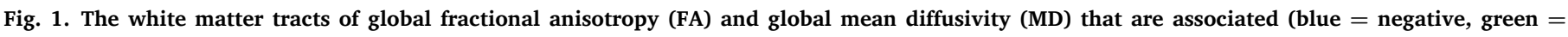

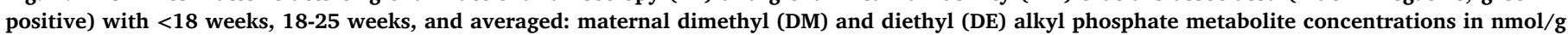

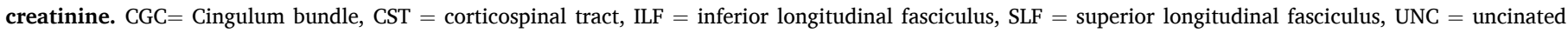

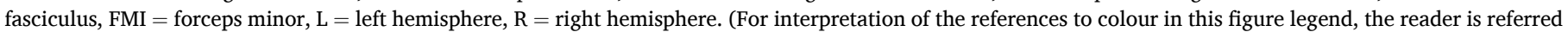
to the Web version of this article.)

subcortical volumes or with white matter microstructure (Table S7). When models were re-run correcting for the potential selection bias using inverse probability weighting, results were similar to the main analyses (Table S8). Of note, a 10-fold increase in averaged DAP metabolite concentrations was significantly associated with a $0.50(95 \%$ $\mathrm{CI}=-0.89,-0.11$ ) decrease in thalamus volume, but the result did not survive the multiple testing correction. Finally, the results in which we additionally adjusted for maternal diet were similar to the main results (Table S9).

\section{Discussion}

In this population-based study, we observed that OP pesticide metabolite concentrations measured during pregnancy were not associated with brain morphological measures including brain volumes, cortical thickness, and cortical surface area in pre-adolescents at 9-12 years of age. However, we showed that higher prenatal exposure to OP pesticides, in particular during early- and mid-pregnancy, was associated with lower FA and higher MD, generally considered as indicators 
for atypical white matter microstructure. When we explored the specific white matter tracts, we observed that OP pesticide exposure was associated with projection, association, limbic system, and callosal fibers.

Although prior studies have noted the importance of the use of neuroimaging tools to address existing research gaps by identifying structural neurotoxic effects of prenatal OP pesticide exposure on the brain (Rauh and Margolis, 2016), only one small epidemiological study has investigated this research question (Rauh et al., 2012). This study observed that prenatal exposure to chlorpyrifos, which devolves into the DE metabolites diethylphosphate and diethylthiophosphate (Sudakin and Stone, 2011), was predictive of enlargement of the cortical surface in several areas including superior and middle temporal gyrus, post-central gyrus, superior frontal gyrus, cuneus and precuneus, and gyrus rectus (Rauh et al., 2012). Furthermore, increased exposure was associated with lower cortical thickness of frontal, temporal, and parietal regions. In contrast to these findings, no evidence of an association with cortical surface area and cortical thickness was observed in our study. The inconsistency in the results may be explained by the differences in OP pesticide exposure, in exposure assessment methodology, or in study populations. Rauh et al. (2012) measured a single OP pesticide chlorpyrifos in cord blood, while in our study we measured DAP metabolites at early-, mid-, and late-pregnancy in urine as a biomarker of OP pesticide exposure. DAP metabolites provide non-specific data about the total exposure to several OP pesticides instead of the exposure to a single OP pesticide. Mothers in the current study were most likely exposed to a combination of different $\mathrm{OP}$ pesticides that also produce $\mathrm{DE}$ metabolites. Of all the insecticides that were applied in 2004 in the Netherlands, $32 \%$ were OP pesticides that produces DM metabolites and only $1 \%$ were pesticides that generate DE metabolites (CBS, 2020). Of the latter, the OP pesticide chlorpyrifos accounted for $1 / 3$ of the total generated DE metabolites. This may suggest that exposure to chlorpyrifos may have been lower in our population. However, between 2004 and 2006 OP pesticide residues of chlorpyrifos coming from importation have been detected on tested vegetables and fruits (ChemKap, 2017). Of note, DAP metabolite concentrations in this study are higher compared to most other birth-cohort studies (van den Dries et al., 2018). Other differences between the studies relate to socio-economic status, as the population in the previous study was socially disadvantaged. It is conceivable that in these populations unmeasured background risk factors related to both chlorpyrifos exposure and brain morphology might lead to potential residual confounding.

To our knowledge, this is the first epidemiological study that investigated prenatal exposure to $\mathrm{OP}$ pesticides and white matter microstructure. In preadolescents aged 9-12 years, the development of many white matter tracts, such as projection of the prefrontal cortex, is still ongoing (Lebel et al., 2019). Altered maturation of white matter microstructure might therefore result in neurodevelopmental problems with long-term clinical implications. Studies have found that altered white matter microstructure is associated with impaired cognition, behavior problems, and neurodevelopmental disorders (Lebel and Deoni, 2018). We observed that increased OP pesticide exposure during pregnancy was associated with lower FA and higher MD of white matter, and that the direction of the associations was consistent across most specific tracts. To help interpret these results we calculated the association of child age with white matter microstructure, as age is a robust determinant of the latter. A one-year increase in age was associated with $0.88(95 \% \mathrm{CI}=0.35,1.42)$ increase in global FA and a $0.10(95 \% \mathrm{CI}=$ $-0.16,-0.05)$ decrease in global MD. This implies that, for example, a 10 -fold increase in averaged DM concentrations during pregnancy has a similar effect as being 1.1 years younger in terms of white matter microstructure.

Global FA and MD are indicators of white matter microstructure (Alexander et al., 2007). FA describes the propensity for enhanced water diffusion in the white matter tracts whereas MD expresses the scale of average water diffusion in every direction within brain tissue (Alexander et al., 2007). A lower FA and higher MD can be a result of several reasons including lower packing of axons, higher membrane permeability, disturbance of internal axonal structure, and decreased myelination (Lebel et al., 2019). Animal studies also observed similar associations in white matter microstructure in relationship with exposure to OP pesticides. Prenatally chlorpyrifos exposed guinea pigs had lower FA and higher MD within the corpus callosum and the amygdala and rats postnatal (day 1 until day 6) exposed to chlorpyrifos had a decreased expression of the myelin-associated glycoprotein in the brain which is crucial for the preservation of the mature myelinated unit (Betancourt et al., 2006; Mullins et al., 2015). Moreover, chlorpyrifos exposure reduces the polymerization of tubulin (Grigoryan and Lockridge, 2009). Tubulin is a protein which plays an important role in the creation of microtubules which are needed for the preservation of the structural and functional integrity of axons (Grigoryan and Lockridge, 2009; Terry, 2012). In our study we further found an association between OP pesticide exposure and white matter microstructure specific tracts present in projection, association, limbic system, and callosal fibers. Further work is required to confirm our observed association between prenatal OP pesticide exposure and altered white matter microstructure in children.

We observed that the first and second trimester $(<18$ weeks, and 18-25 weeks) OP pesticide exposure were driving the association with lower FA and higher MD. White matter growth starts in early gestation and myelination begins in the second trimester (Dubois et al., 2014; Huang et al., 2009; Rice and Barone, 2000). OP pesticide exposure has been shown to disrupt the expression of genes and proteins important for the myelination (Slotkin and Seidler, 2007). During the second trimester, the development of white matter is especially dependable on signaling pathways such as extracellular ligands, secreted molecules, and transcriptional regulations (Emery, 2010). Thus, OP pesticide exposure may alter the courses of later brain development by influencing axonal growth adhering and group formation and white matter myelination via gene expression alteration.

This study has several limitations. First, urinary DAP metabolite concentrations provide information regarding the joint exposure to multiple OP pesticides instead of providing specific information regarding the exact OP pesticide exposure (Duggan et al., 2003; Margariti et al., 2007; Wessels et al., 2003). It is therefore unknown to which specific OP parent pesticide(s) our study population was exposed. However, the use of DAP metabolites as biomarkers of OP pesticides is also a strength because it allows for the identification and comparison of OP pesticide exposure levels within and between study populations (Bravo et al., 2004). Second, DAP metabolites are characterized by a short half-life and are excreted in urine within one or two days. This implies that the biomarker concentrations may differ from day-to-day within each subject as a consequence of variable contact with exposure sources (e.g., variable diet patterns) and result in (within-subject) temporal variability (Lu et al., 2008; Needham, 2005). Although we included 3 urine spot samples which is more frequent than most other studies exploring the association between prenatal OP pesticide exposure and neurodevelopment, it would be preferable to collect more urine specimens during pregnancy to reduce the measurement error and attenuation bias caused by the within-subject variability (Perrier et al., 2016). Third, this study was restricted by the nonappearance of information on possible residential pesticide use by the participant, another household member, or a professional exterminator. Participants in this study might have been exposed through the use of residential products which may contain OP pesticides such as insecticides for the lawn and garden (e.g., emulsifiable concentrate), insecticides for house plants, residential pest products (e.g., fly control insecticides and moth killer cassettes), and flea products for pets. Although the use of products that contain OP pesticides is unlikely to confound the association between prenatal OP pesticide exposure and brain morphology, such information would be helpful in determining the exact sources of the exposure. The Generation R Study is representative of an urban population of which the exposure to OP pesticides most likely occurs through diet (van den Dries et al., 2018). The results of this study may therefore not be fully 
generalizable to semi-urban and rural areas in the Netherlands where the source of OP pesticide exposure could be different. Finally, this study was limited by the absence of information on exposure to other types of pesticides. While we included many possible confounders in our analyses, we cannot eliminate the existence of potential residual confounding in this study as a consequence of unidentified background risk factors that are predictive of OP pesticide exposure and brain development.

The present study has a number of strengths. This study has a large sample size and the availability of many potential confounders such as the IQ of the mother and socio-economic factors. Further, the scanning procedure in which all brains were scanned using the same MRI scanner and software to reduce potential measurement error is another strength.

In conclusion, prenatal OP pesticide exposure was not associated with brain volumes, cortical thickness, and cortical surface area in preadolescents aged 9-12 years. However, we found that prenatal OP pesticide exposure was associated with lower FA and higher MD of white matter, and that early and mid-pregnancy exposure were driving these associations. These findings suggest that prenatal exposure to worldwide commonly applied OP pesticides may alter normal white matter microstructure development in children, which could have consequences for normal neurodevelopment. Besides structural brain changes, functional brain alteration may also provide opportunities to deepen the understanding of the effects of prenatal exposure to OP pesticides on the brain, as a recent study has done (Sagiv et al., 2019). Future studies on brain imaging are warranted to reproduce our findings, as well as to investigate the mediating role of the structural and functional brain alterations in the association between prenatal exposure to $\mathrm{OP}$ pesticides and neuropsychological development. If the findings of this study are confirmed, public health policies that aim towards stricter regulation and control of OP pesticides application should be implemented both in Europe and worldwide.

\section{Authorship contribution statement}

Michiel A. van den Dries: Conceptualisation; Methodology; Formal analysis; Writing - original draft; Writing - review \& editing; Visualisation. Sander Lamballais: Formal analysis; Software; Writing - review \& editing. Hanan El Marroun: Writing - review \& editing. Anjoeka Pronk: Writing - review \& editing; Funding acquisition. Suzanne Spaan: Writing - review \& editing. Kelly K. Ferguson: Writing - review \& editing. Matthew P. Longnecker: Writing - review \& editing; Funding acquisition. Henning Tiemeier: Writing - review \& editing; Resources; Funding acquisition. Mònica Guxens: Conceptualisation; Methodology; Writing - original draft; Writing - review \& editing; Resources; Supervision; Project administration.

\section{Funding}

This research received financial support from the intramural research program of the National Institute of Environmental Health Sciences, National Institutes of Health (Grant\# HHSN273201500003C). The general design of the Generation R Study is made possible by financial support from the Erasmus Medical Center, Rotterdam; the Erasmus University Rotterdam; Netherlands Organization for Health Research and Development (ZonMw); the Netherlands Organization for Scientific Research (NWO); and the Ministry of Health, Welfare and Sport. Neuroimaging was supported by the ZonMw TOP project no. 91211021, and super computing computations for imaging processing were supported by the NWO Physical Sciences Division (Exacte Wetenschappen) and SURFsara (Cartesius compute cluster, https://www.surf.nl). Henning Tiemeier was supported by a grant of the Netherlands Organization for Scientific Research (NWO grant No. 024.001.003, Consortium on Individual Development, and NWO/ ZonMW grant 016.VICI.170.200). Mònica Guxens is funded by a Miguel Servet fellowship (MS13/00054, CPII18/00018) awarded by the
Spanish Institute of Health Carlos III. Monica Guxens acknowledges support from the Spanish Ministry of Science and Innovation and State Research Agency through the "Centro de Excelencia Severo Ochoa 2019-2023" Program (CEX2018-000806-S), and support from the Generalitat de Catalunya through the CERCA Programme. Dr. Hanan El Marroun was supported by Stichting Volksbond Rotterdam, the Dutch Brain Foundation (De Hersenstichting, project number GH2016.2.01) and the European Union's Horizon 2020 research and innovation programme (grant agreement No. 733206 LifeCycle). Funders for this research had no participation in the design and conduct of the study.

\section{Ethics approval and consent to participate}

Human subjects review for the procedure of this study was carried out and approved by the Medical Ethics Committee of the Erasmus Medical Center, Rotterdam (IRB Registration no.: IRB00001482, MEC2012-165, MEC-2007-413, MEC, 217.595/2002/202, and MEC 198.782.2001.31). Written informed consent for the children and mothers was provided by the mothers.

\section{Declaration of competing interest}

The authors declare that they have no known competing financial interests or personal relationships that could have appeared to influence the work reported in this paper.

\section{Acknowledgement}

We would like to acknowledge all the partners involved in the Generation R Study which includes the Erasmus Medical Center, Rotterdam, the Faculty of Social Sciences of the Erasmus University Rotterdam, Rotterdam Homecare Foundation and Stichting Trombosedienst \& Artsenlaboratorium Rijnmond. Further, the authors sincerely appreciate Dr. Frank Pierik effort for arranging the basis for the NIEHS-TNOErasmus University partnership.

\section{Appendix A. Supplementary data}

Supplementary data to this article can be found online at https://doi. org/10.1016/j.envres.2020.110047.

\section{References}

Alexander, A.L., Lee, J.E., Lazar, M., Field, A.S., 2007. Diffusion tensor imaging of the brain. Neurotherapeutics 4, 316-329.

Betancourt, A.M., Burgess, S.C., Carr, R.L., 2006. Effect of developmental exposure to chlorpyrifos on the expression of neurotrophin growth factors and cell-specific markers in neonatal rat brain. Toxicol. Sci. 92, 500-506.

Bradman, A., Barr, D.B., Claus Henn, B.G., Drumheller, T., Curry, C., Eskenazi, B., 2003. Measurement of pesticides and other toxicants in amniotic fluid as a potential biomarker of prenatal exposure: a validation study. Environ. Health Perspect. 111, 1779-1782.

Bravo, R., Caltabiano, L.M., Weerasekera, G., Whitehead, R.D., Fernandez, C., Needham, L.L., et al., 2004. Measurement of dialkyl phosphate metabolites of organophosphorus pesticides in human urine using lyophilization with gas chromatography-tandem mass spectrometry and isotope dilution quantification, 14, 249.

CBS. 2020.https://opendata.cbs.nl/statline/\#/CBS/nl/dataset/37606/table? $\mathrm{dl}=3513 \mathrm{D}$ (Accessed 20 March 2020).

ChemKap, 2017. Agricultural Products Quality Programme Database Chemkap.

Cook, P.A., Bai, Y., Nedjati-Gilani, S., Seunarine, K.K., Hall, M.G., Parker, G.J., et al., 2006. Camino: open-source diffusion-mri reconstruction and processing. In: Proceedings of the 14th Scientific Meeting of the International Society for Magnetic Resonance in Medicine, 2759, p. 2759. Seattle WA, USA.

Costa, L.G., Giordano, G., Guizzetti, M., Vitalone, A., 2008. Neurotoxicity of pesticides: a brief review. Front. Biosci. 13, 1240-1249.

de Groot, M., Ikram, M.A., Akoudad, S., Krestin, G.P., Hofman, A., van der Lugt, A., et al., 2015. Tract-specific white matter degeneration in aging: the rotterdam study. Alzheimer's Dementia 11, 321-330.

Dennis, E.L., Thompson, P.M., 2013. Typical and atypical brain development: a review of neuroimaging studies. Dialogues Clin. Neurosci. 15, 359-384. 
Dubois, J., Dehaene-Lambertz, G., Kulikova, S., Poupon, C., Hüppi, P.S., HertzPannier, L., 2014. The early development of brain white matter: a review of imaging studies in fetuses, newborns and infants. Neuroscience 276, 48-71.

Duggan, A., Charnley, G., Chen, W., Chukwudebe, A., Hawk, R., Krieger, R.I., et al., 2003. Di-alkyl phosphate biomonitoring data: assessing cumulative exposure to organophosphate pesticides. Regul. Toxicol. Pharmacol. 37, 382-395.

Eaton, D.L., Daroff, R.B., Autrup, H., Bridges, J., Buffler, P., Costa, L.G., et al., 2008. Review of the toxicology of chlorpyrifos with an emphasis on human exposure and neurodevelopment. Crit. Rev. Toxicol. 38, 1-125.

Emery, B., 2010. Regulation of oligodendrocyte differentiation and myelination. Science 330, 779-782.

Pesticides database. 2020. https://eceuropaeu/food/plant/pesticides/eu-pesticides -database/public/?event=homepage\&language $=\mathrm{EN}$.

Fenske, R.A., Lu, C., Barr, D., Needham, L., 2002. Children's exposure to chlorpyrifos and parathion in an agricultural community in central Washington state. Environmental Health Perspect. 110, 549-553.

Gilmore, J.H., Knickmeyer, R.C., Gao, W., 2018. Imaging structural and functional brain development in early childhood. Nat. Rev. Neurosci. 19, 123-137.

Grigoryan, H., Lockridge, O., 2009. Nanoimages show disruption of tubulin polymerization by chlorpyrifos oxon: implications for neurotoxicity. Toxicol. Appl. Pharmacol. 240, 143-148.

Huang, H., Xue, R., Zhang, J., Ren, T., Richards, L.J., Yarowsky, P., et al., 2009. Anatomical characterization of human fetal brain development with diffusion tensor magnetic resonance imaging. J. Neurosci. 29, 4263-4273.

Julien, R., Adamkiewicz, G., Levy, J.I., Bennett, D., Nishioka, M., Spengler, J.D., 2007. Pesticide Loadings of Select Organophosphate and Pyrethroid Pesticides in Urban Public Housing, vol. 18, p. 167.

Kooijman, M.N., Kruithof, C.J., van Duijn, C.M., Duijts, L., Franco, O.H., van Ijzendoorn, M.H., et al., 2016. The generation r study: design and cohort update 2017. Eur. J. Epidemiol. 31, 1243-1264.

Lebel, C., Deoni, S., 2018. The development of brain white matter microstructure. Neuroimage 182, 207-218.

Lebel, C., Treit, S., Beaulieu, C., 2019. A review of diffusion mri of typical white matter development from early childhood to young adulthood. NMR Biomed. 32, e3778.

Lu, C., Fenske, R.A., Simcox, N.J., Kalman, D., 2000. Pesticide exposure of children in an agricultural community: evidence of household proximity to farmland and take home exposure pathways. Environ. Res. 84, 290-302.

Lu, C., Kedan, G., Fisker-Andersen, J., Kissel, J.C., Fenske, R.A., 2004. Multipathway organophosphorus pesticide exposures of preschool children living in agricultural and nonagricultural communities. Environ. Res. 96, 283-289.

Lu, C., Barr, D.B., Pearson, M.A., Waller, L.A., 2008. Dietary intake and its contribution to longitudinal organophosphorus pesticide exposure in urban/suburban children. Environ. Health Perspect. 116, 537-542.

Lubczyńska, M.J., Muetzel, R.L., El Marroun, H., Basagaña, X., Strak, M., Denault, W., Jaddoe, V.W.V., Hillegers, M., Vernooij, M., Hoek, G., White, T., Brunekreef, B., Tiemeier, H., Guxens, M., 2020. Exposure to Air Pollution during Pregnancy and Childhood, and White Matter Microstructure in Preadolescents. Environ. Health Perspect. 128 (2) https://doi.org/10.1289/EHP4709.

Mahajan, R., Chandel, S., Chatterjee, S., 2019. Environmental fate of organophosphate residues from agricultural soils to fresh farm produce: microbial interventions for sustainable bioremediation strategies. In: Microbes and Enzymes in Soil Health and Bioremediation. Springer, pp. 211-224.

Margariti, M.G., Tsakalof, A.K., Tsatsakis, A.M., 2007. Analytical methods of biological monitoring for exposure to pesticides: recent update. Ther. Drug Monit. 29, $150-163$.

Mizuno, Y., Kagitani-Shimono, K., Jung, M., Makita, K., Takiguchi, S., Fujisawa, T.X., et al., 2019. Structural brain abnormalities in children and adolescents with comorbid autism spectrum disorder and attention-deficit/hyperactivity disorder. Transl. Psychiatry 9, 332.

Muetzel, R.L., Mous, S.E., van der Ende, J., Blanken, L.M.E., van der Lugt, A., Jaddoe, V. W.V., et al., 2015. White matter integrity and cognitive performance in school-age children: a population-based neuroimaging study. Neuroimage 119, 119-128.

Muetzel, R.L., Blanken, L.M.E., van der Ende, J., El Marroun, H., Shaw, P., Sudre, G., et al., 2017. Tracking brain development and dimensional psychiatric symptoms in children: a longitudinal population-based neuroimaging study. Am. J. Psychiatr. 175, 54-62.

Muetzel, R.L., Mulder, R.H., Lamballais, S., Cortes Hidalgo, A.P., Jansen, P., Güroğlu, B., et al., 2019. Frequent bullying involvement and brain morphology in children. Front. Psychiatr. 10

Mullins, R.J., Xu, S., Pereira, E.F.R., Pescrille, J.D., Todd, S.W., Mamczarz, J., et al., 2015. Prenatal exposure of Guinea pigs to the organophosphorus pesticide chlorpyrifos disrupts the structural and functional integrity of the brain. Neurotoxicology 48, 9-20.
Needham, L.L., 2005. Assessing exposure to organophosphorus pesticides by biomonitoring in epidemiologic studies of birth outcomes. Environ. Health Perspect. 113, 494-498.

Perrier, F., Giorgis-Allemand, L., Slama, R., Philippat, C., 2016. Within-subject pooling of biological samples to reduce exposure misclassification in biomarker-based studies. Epidemiology 27, 378-388.

Prieler, J., 2003. Raven's Advanced Progressive Matrices. Schufried, Mödling, Austria.

R core Team, 2015. A Language and Environment for Statistical Computing. R Foundation for Statistical Computing, Vienna, austria.

Rauh, V.A., Perera, F.P., Horton, M.K., Whyatt, R.M., Bansal, R., Hao, X., et al., 2012. Brain anomalies in children exposed prenatally to a common organophosphate pesticide. Proc. Natl. Acad. Sci. U. S. A. 109, 7871-7876.

Rauh, V.A., Margolis, A.E., 2016. Research review: environmental exposures, neurodevelopment, and child mental health - new paradigms for the study of brain and behavioral effects. JCPP (J. Child Psychol. Psychiatry) 57, 775-793.

Rice, D., Barone, S., 2000. Critical periods of vulnerability for the developing nervous system: evidence from humans and animal models. Environ. Health Perspect. 108, 511-533.

Roy, T.S., Seidler, F.J., Slotkin, T.A., 2004. Morphologic effects of subtoxic neonatal chlorpyrifos exposure in developing rat brain: regionally selective alterations in neurons and glia. Dev. Brain Res. 148, 197-206.

Roy, T.S., Sharma, V., Seidler, F.J., Slotkin, T.A., 2005. Quantitative morphological assessment reveals neuronal and glial deficits in hippocampus after a brief subtoxic exposure to chlorpyrifos in neonatal rats. Dev. Brain Res. 155, 71-80.

Sagiv, S.K., Bruno, J.L., Baker, J.M., Palzes, V., Kogut, K., Rauch, S., et al., 2019. Prenatal exposure to organophosphate pesticides and functional neuroimaging in adolescents living in proximity to pesticide application. Proc. Natl. Acad. Sci. Unit. States Am. $116,18347$.

Sapbamrer, R., Hongsibsong, S., 2019. Effects of prenatal and postnatal exposure to organophosphate pesticides on child neurodevelopment in different age groups: a systematic review. Environ. Sci. Pollut. Control Ser. 26, 18267-18290.

Savy, C.Y., Fitchett, A.E., Blain, P.G., Morris, C.M., Judge, S.J., 2018. Gene expression analysis reveals chronic low level exposure to the pesticide diazinon affects psychological disorders gene sets in the adult rat. Toxicology 393, 90-101.

Slotkin, T.A., Seidler, F.J., 2007. Comparative developmental neurotoxicity of organophosphates in vivo: transcriptional responses of pathways for brain cell development, cell signaling, cytotoxicity and neurotransmitter systems. Brain Res. Bull. 72, 232-274.

Slotkin, T.A., Bodwell, B.E., Levin, E.D., Seidler, F.J., 2008. Neonatal exposure to low doses of diazinon: long-term effects on neural cell development and acetylcholine systems. Environ. Health Perspect. 116, 340-348.

Steenweg-de Graaff, J., Roza, S.J., Steegers, E.A., Hofman, A., Verhulst, F.C., Jaddoe, V. W., et al., 2012. Maternal folate status in early pregnancy and child emotional and behavioral problems: the generation r study. Am. J. Clin. Nutr. 95, 1413-1421.

Sudakin, D.L., Stone, D.L., 2011. Dialkyl phosphates as biomarkers of organophosphates: the current divide between epidemiology and clinical toxicology. Clin. Toxicol. 49, $771-781$.

Terry, A.V., 2012. Functional consequences of repeated organophosphate exposure: potential non-cholinergic mechanisms. Pharmacol. Ther. 134, 355-365.

Textor, J., van der Zander, B., Gilthorpe, M.S., Liśkiewicz, M., Ellison, G.T.H., 2017. Robust causal inference using directed acyclic graphs: the r package 'dagitty'. Int. J. Epidemiol. 45, 1887-1894.

Valcke, M., Samuel, O., Bouchard, M., Dumas, P., Belleville, D., Tremblay, C., 2006. Biological monitoring of exposure to organophosphate pesticides in children living in peri-urban areas of the province of quebec, Canada. Int. Arch. Occup. Environ. Health 79, 568-577.

van Buuren, S., Groothuis-Oudshoorn, C., 2011. Mice: Multivariate Imputation by Chained Equations in R.

van den Dries, M.A., Pronk, A., Guxens, M., Spaan, S., Voortman, T., Jaddoe, V.W., et al., 2018. Determinants of organophosphate pesticide exposure in pregnant women: a population-based cohort study in The Netherlands. Int. J. Hyg Environ. Health 221, 489-501.

van den Dries, M.A., Guxens, M., Pronk, A., Spaan, S., El Marroun, H., Jusko, T.A., et al., 2019. Organophosphate pesticide metabolite concentrations in urine during pregnancy and offspring attention-deficit hyperactivity disorder and autistic traits. Environ. Int. 131, 105002.

Wessels, D., Barr, D.B., Mendola, P., 2003. Use of biomarkers to indicate exposure of children to organophosphate pesticides: implications for a longitudinal study of children's environmental health. Environmental Health Perspect. 111, 1939-1946.

Whyatt, R.M., Barr, D.B., Camann, D.E., Kinney, P.L., Barr, J.R., Andrews, H.F., et al., 2003. Contemporary-use pesticides in personal air samples during pregnancy and blood samples at delivery among urban minority mothers and newborns. Environ. Health Perspect. 111, 749-756. 